\title{
QUEEN'S
UNIVERSITY
BELFAST
}

\section{How to blow up a massive star}

Müller , B., \& Smartt, S. (2017). How to blow up a massive star. Astronomy \& Geophysics, 58(2), 2.32-2.37. https://doi.org/10.1093/astrogeo/atx061

Published in:

Astronomy \& Geophysics

Document Version:

Publisher's PDF, also known as Version of record

Queen's University Belfast - Research Portal:

Link to publication record in Queen's University Belfast Research Portal

\section{General rights}

Copyright for the publications made accessible via the Queen's University Belfast Research Portal is retained by the author(s) and / or other copyright owners and it is a condition of accessing these publications that users recognise and abide by the legal requirements associated with these rights.

Take down policy

The Research Portal is Queen's institutional repository that provides access to Queen's research output. Every effort has been made to ensure that content in the Research Portal does not infringe any person's rights, or applicable UK laws. If you discover content in the Research Portal that you believe breaches copyright or violates any law, please contact openaccess@qub.ac.uk. 


\section{How to blow up a massive star}

\section{Bernhard Müller and Stephen \\ Smartt explain how simulations combined with astronomical observations are shaping a more coherent picture of the explosions of massive stars.}

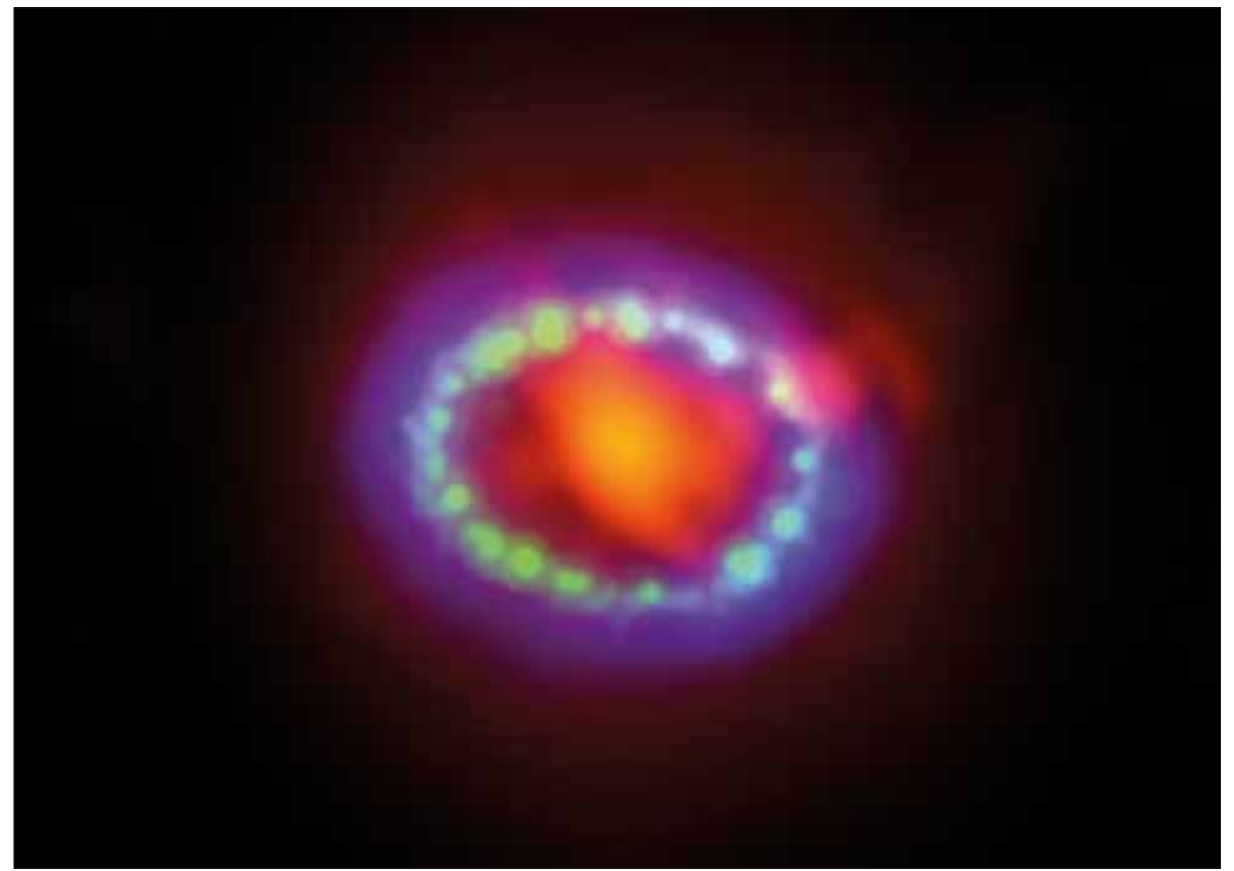

1 Supernova remnant SN 1987a in false colour. ALMA data (red) show newly formed dust; Hubble (green) and Chandra (blue) data show where the expanding shock wave meets an outer ring of dust and gas. (ALMA [ESO/NAOJ/NRAO]/A Angelich. Visible: NASA/ESA Hubble. X-ray: NASA Chandra)

upernovae have a peculiar place in the history of astronomy. They are the only transient phenomena in the sky outside the solar system with a documented history of observations by the naked eye for almost two millennia: prominent examples include the supernova of 1054 that made the Crab Nebula, and those of 1572 and 1604 - now named after Tycho Brahe and Johannes Kepler - which shook the medieval belief in an immutable celestial sphere. Yet it was not until the 20th century that we began to grasp their true nature and realized - starting with the work of Baade \& Zwicky (1934b) - that they are violent stellar explosions.

The energy budget of supernovae is impressive indeed: they can outshine their host galaxy for weeks and the ejected material reaches several $1000 \mathrm{~km} \mathrm{~s}^{-1}$, implying an explosion energy of $\sim 10^{51} \mathrm{erg}$. In view of this enormous energy, Baade \& Zwicky (1934a) suggested that some supernovae are associated with gravitational collapse of a massive star to a neutron star - only two years after the discovery of the neutron and more than 30 years before the first neutron star was observed.

As stellar evolution and nuclear astrophysics matured, the details of Baade and Zwicky's far-sighted idea - what we now call "core-collapse supernovae"- were worked out. They occur in stars that begin their lives with a mass of more than $8 \mathrm{M}_{\odot}$ (solar masses). In this mass range, stars can ignite advanced burning stages (carbon, neon, oxygen and silicon burning) after processing hydrogen and then helium in their cores. Eventually, these stars form an onion shell structure with a core composed 
2 Sketch of the neutrino-driven supernova explosion mechanism. After collapse, the iron core has formed a proto-neutron star (orange). The proto-neutron star cools by the emission of neutrinos that diffuse out on a timescale of about 10 seconds from the proto-neutron star interior. Infalling matter that settles on the proto-neutron star surface as an "atmosphere" (grey) also readily emits neutrinos. Outside the "gain radius" (dotted circle), neutrino heating through reabsorption dominates over cooling and drives convective overturn. The shock can also develop an oscillatory instability ("SASI"). Neutrino cooling drives convection inside the proto-neutron star as well.

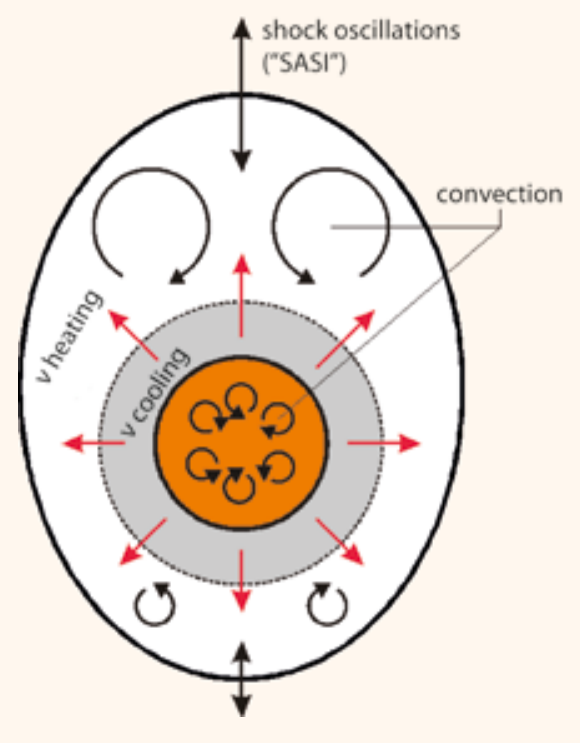

(radius, neutrino energy and one direction angle). Moreover, there are further technical complications, for example one needs to model the transport of neutrinos in curved spacetime because of the strong gravity of the proto-neutron star.

It therefore took several decades after the original idea of Colgate \& White (1966) and the birth of general relativistic kinetic theory (Lindquist 1966) for spherically symmetric (1D) simulations to become sufficiently accurate to model the hydrodynamics and neutrino transport in the core of a supernova. Modern 1D simulations solve the Boltzmann equation for neutrinos and have now convincingly established that the "neutrino-driven mechanism" does not work under the assumption of spherical symmetry (Liebendoerfer et al. 2001, Rampp \& Janka 2000) for most massive stars. Only the lightest supernova progenitors form an exception. For stars with initial mass $8-10 \mathrm{M}_{\odot}$, the density declines so rapidly outside the iron core that the pre-shock ram pressure of the infalling shells plummets very quickly and allows neutrino heating to drive a low-energy explosion of $\sim 10^{50} \mathrm{erg}$ (Kitaura et al. 2006). There are several direct detections of the progenitor stars of lowenergy supernovae and they are consistent with being red supergiants in the $8-12 \mathrm{M}_{\odot}$ mass range, such as those for $\mathrm{SN} 2005 \mathrm{cs}$, SN2009md and SN2008bk (Fraser et al. 2011, Maund et al. 2005, 2014).

\section{Multidimensional models}

The solution for the supernova explosion problem for more massive stars lies in breaking spherical symmetry. As was pointed out by several authors in the 1990s (e.g. Herant et al. 1992, Burrows et al. 1995), multidimensional effects facilitate neutrino-powered explosions in various ways. Convective overturn, driven by strong neutrino heating close to the proto-neutron star, transports hot material outwards,

\section{Simulating the mechanism}

Tapping the gravitational energy directly after collapse might appear to be the simplest solution. Once the inner part of the iron scattering and propagation of neutrinos the core of the supernova using numerical simulations. Because the neutrinos fall out of thermal equilibrium with the stellar matter in the proto-neutron star surface, this is a problem in kinetic theory. Even if we assume the collapse and explosion as perfectly spherical, we are still left with a three-dimensional problem in phase space 

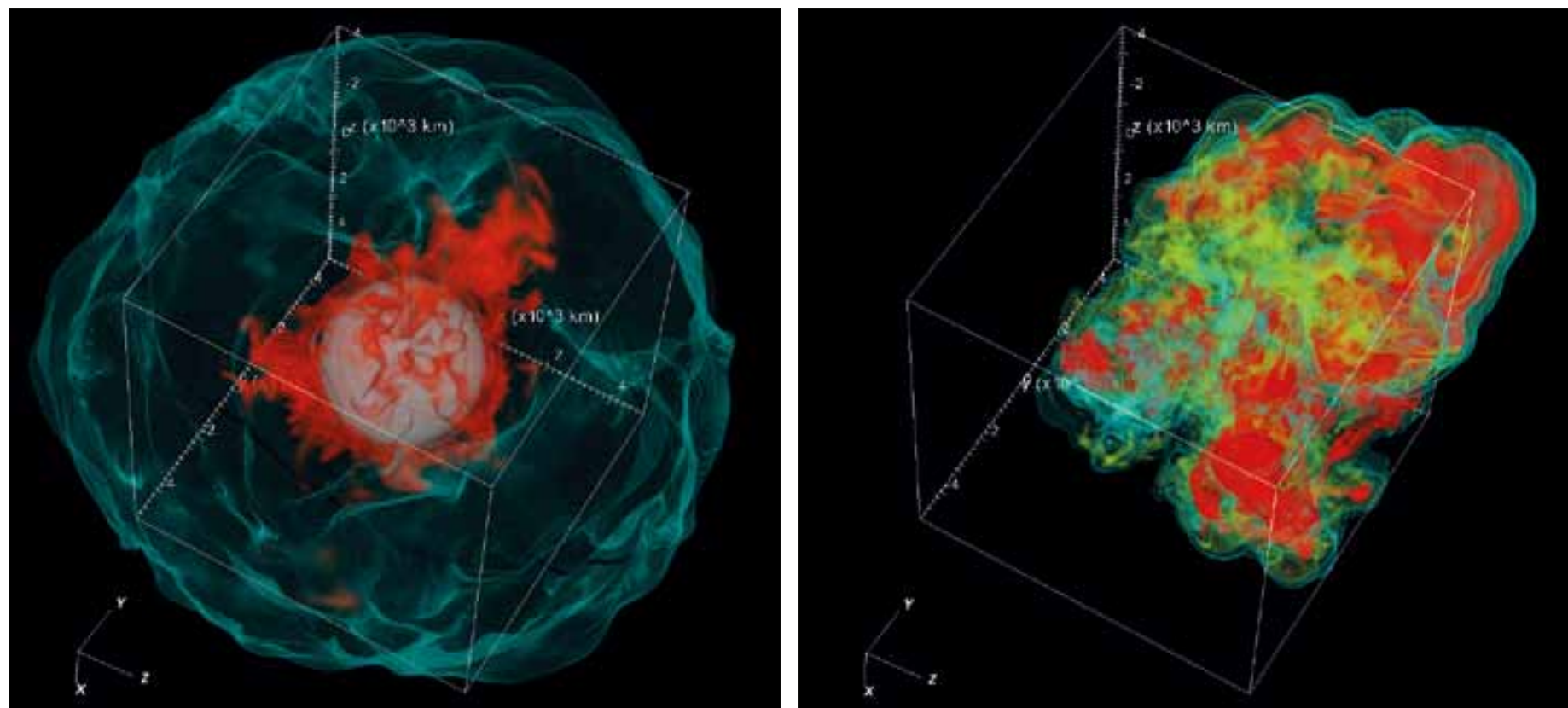

3 (Left) Convection during oxygen shell burning in a 3D progenitor model of an $18 \mathrm{M}_{\odot}$ star. The snapshot shows plumes of upwelling silicon-rich ashes (red) in the $2 o^{\prime}$ clock and $9 o^{\prime}$ clock directions and a weaker plume in the $5 o^{\prime}$ clock direction. The boundary of the oxygen burning shell (cyan, transparent) is corrugated by convective overturn motions. The inert silicon/iron core is shown in grey. (Right) Volume rendering of the entropy in the corresponding supernova explosion model, $1.2 \mathrm{~s}$ after the collapse of the iron core to a neutron star. Hot neutrino-heated matter (red) is ejected asymmetrically from the young neutron star, preferentially in the direction of the the strongest silicon-rich plume at $2 o^{\prime}$ clock from the previous epoch of oxygen shell burning.

thereby increasing the thermal pressure behind the shock. As a result, the shock expands, which in turn increases the mass that can be heated by neutrinos. The effective pressure exerted by convective bubbles bouncing against the shock has a similar effect, as does another instability - dubbed SASI, the "standing accretion shock instability" (Blondin et al. 2003) - that result in large-scale oscillatory shock motions.

For a long time, multidimensional models of these phenomena had to compromise severely on the accuracy of the neutrino transport. Axisymmetric (2D) simulations with an accuracy on a par with their 1D counterparts became computationally affordable about a decade ago, and many of them developed the coveted explosions (Janka 2012). As a solution of the supernova problem, this proved a little premature. The step to 3D simulations held a surprise: 3D models are now generally found to be slightly more reluctant to explode than their 2D counterparts. Indeed, the first 3D models with rigorous neutrino transport failed to explode (Hanke et al. 2013), contrary to the behaviour of 2D simulations. The main reason for the different behaviour of the 2D and 3D models seems to lie in a peculiarity of 2D turbulent flows, which tend to form large-scale eddies that dissipate the turbulent kinetic energy less efficiently than in three dimensions.

Because many massive stars are observed to explode at least in the mass range $8-17 \mathrm{M}_{\odot}$ (for a review see Smartt 2009, 2015), something was evidently missing in those first unsuccessful 3D simulations. Or perhaps these unsuccessful explosion models targeted progenitor stars that are less likely to explode in reality? Identifying the missing ingredients or which progenitors can explode is time consuming and hard work. Depending on the treatment of the neutrino transport, a single model requires up to about 50 million CPU hours and takes months on massively parallel supercomputers. Nevertheless, several groups have reported successful model explosions in the past two years, e.g. for progenitor stars of $9.6 \mathrm{M}_{\odot}$ (Melson et al. 2015b) and $15 \mathrm{M}_{\odot}$ (Lentz et al. 2015). A number of simulations set out to identify missing physics that could lead to robust 3D explosion models, such as modifications to the neutrino opacities (Melson et al. 2015a) and stellar rotation (Janka et al. 2016).

Another idea for solving the problem of shock revival revisits the structure of the supernova progenitors themselves. Since some of the collapse shells are convective shells (driven by oxygen, neon or silicon burning), the infall of matter onto the shock is not spherically symmetric. Numerical studies based on parametrized multidimension initial conditions (Couch \& Ott 2013, Mueller \& Janka 2015) and analytic works (Abdikamalov et al. 2016) showed that the infalling non-spherical perturbations couple to convection and the SASI and can boost them to an extent that neutrinodriven explosions become significantly easier. This scenario of a "perturbationaided" neutrino-driven mechanism is particularly attractive because it does not rely on unknown physics or on tweaked stellar evolution models (e.g. achieving rapid rotation). The presence of convective shells is predicted naturally by stellar evolution models, albeit based on an effective 1D model for convection known as mixinglength theory.

To test the "perturbation-aided" neutrino-driven mechanism, Couch et al. (2015) and Müller et al. (2016b) recently conducted 3D simulations of shell burning up to the onset of collapse for $15 \mathrm{M}_{\odot}$ and
18 $\mathrm{M}$ progenitors. These massively parallel simulations show convective updrafts and downdrafts with velocities of several $100 \mathrm{~km} \mathrm{~s}^{-1}$, which are organized in a few larger eddies

if the convection zone is sufficiently deep (figure 3). Upon collapse, the updrafts collapse more slowly and, in a manner of speaking, offer less resistance to the shock (smaller ram pressure). In the case of the 3D progenitor model of an $18 \mathrm{M}_{\odot}$ star of Müller et al. (2016b), this leads to shock revival about $250 \mathrm{~ms}$ after core bounce, whereas a simulation with a spherically symmetric progenitor model failed to explode. Analytic estimates suggest that this effect could reduce the amount of required neutrino heating for shock revival by up to a quarter, but it remains to be seen whether sufficiently violent convection is common across supernova progenitors.

All this suggests that 3D supernova simulations are now on track towards understanding shock revival. Undoubtedly many of the ideas formulated in recent years will contribute to the solution to some degree and the current progress would, of 
course, be unthinkable without modern methods for neutrino transport, which build on decades of code development.

Predicting explosion properties by means of multidimensional simulations remains a bigger challenge. The reason for this is that the neutrino-driven engine can operate for a second or more, as long as accretion downflows funnel fresh matter onto the proto-neutron star surface; this keeps the neutrino luminosity and hence the mass outflow rate in neutrino-heated bubbles high. Few simulations can be extended to well above a second after core bounce, and therefore the final explosion energies and the amount and composition of the ejecta remain uncertain. 3D simulation can make firm predictions only for progenitors at the low-mass end, where Melson et al. (2015b) predict an explosion energy of $\sim 10^{50} \mathrm{erg}$ for a $9.6 \mathrm{M}_{\odot}$ progenitor. Self-consistent 2D simulations can be taken well beyond the first second (Müller 2015, Bruenn et al. 2016) and are cheap enough to scan a wide range of progenitor models (Nakamura 2015), but cannot be trusted to predict the energetics of the explosion correctly (Müller 2015).

\section{The explosion landscape}

We are therefore relegated to following other approaches to obtain predictions that can actually be confronted with observations of the explosions themselves, of their progenitors and their compact and gaseous remnants.

To this end, various authors have developed more phenomenological models for predicting supernova explosion properties. Early works from around 2000 (e.g. Fryer \& Kalogera 2001, Heger et al. 2003) that studied the "explodability" of supernova progenitors as a function of their initial mass were based on a simple comparison of the binding energy of the envelope and the explosion energy provided by the neutrinodriven engine, which could not be reliably predicted at that time.

In recent years, several groups have developed better phenomenological supernova models that take the physics of the neutrino-driven mechanism into account, in a simplified form. Various authors have used 1D simulations with artificially enhanced neutrino heating to study the "explodability" of supernova progenitors (O'Connor \& Ott 2011, Ugliano et al. 2012, Ertl et al. 2016, Perego et al. 2015). These studies have estimated the compact remnant mass function and potential observables that can be inferred from light curves and spectra, such as the explosion energy and the amount of ${ }^{56} \mathrm{Ni}$.

The radioactive isotope ${ }^{56} \mathrm{Ni}$ is one of the most important isotopes in astrophysics and is key to understanding supernovae and nucleosynthesis. As the shock from core-collapse shoots through the inner silicon and oxygen layers of the progenitor star, high entropy causes the quasiequilibrium and burning of the alpha-rich gas predominantly to ${ }^{56} \mathrm{Ni}$. The decay of this to stable ${ }^{56} \mathrm{Fe}\left(\right.$ via $\left.{ }^{56} \mathrm{Co}\right)$ powers Type I supernovae and the later phases of Type II supernovae. The mass of ${ }^{56} \mathrm{Ni}$ produced is a direct probe of the explosion just seconds after core collapse and this mass can be measured from the luminosity of supernovae at different stages.

The most advanced and comprehensive study of a population of supernova progenitor and explosion models is that of Sukhbold et al. (2016). These authors ....... tuned their model for the neutrino-driven engine to fit the explosion properties of SN1987A (figure 1), which had an explosion energy of $\sim 1.3 \times 10^{51} \mathrm{erg}$ and produced $0.07 \mathrm{M}_{\odot}$ of ${ }^{56} \mathrm{Ni}$, and those of the $\mathrm{Crab}$ supernova SN1054 at the low-mass end with an explosion energy $\sim 10^{50} \mathrm{erg}$. Based on the results of their 1D simulations, they also compute supernova light curves and study the detailed population-integrated nucleosynthesis from progenitors in the range $9-120 \mathrm{M}_{\odot}$.

Other studies have opted for an analytic framework to study the systematics of neutrino-driven explosions (Fryer et al. 2012, Müller et al. 2016a and, to some extent, Pejcha \& Thompson 2015). Such analytic approaches need to simplify the physics of the neutrino-driven mechanism even more than calibrated 1D simulations, but have two important advantages. First, exploring parameter variations to test the robustness of the predictions is cheap. Secondly, analytic models can approximately account for effects that are missing in 1D simulations, such as convective overturn. Guided by 3D simulations, Müller et al. (2016a), for example, use semi-empirical scaling laws for the neutrino emission and mass outflow rate in neutrino-heated bubbles (compare to figure 3) to estimate how the explosion energy grows due to a cycle of accretion and mass ejection over timescales of seconds, something which is beyond 1D models by construction.

Reassuringly, the different phenomenological approaches to the supernova explosion model complement each other well. There is broad qualitative agreement about trends in the explodability of supernova progenitors of solar metallicity. Despite variations in detail, recent theoretical studies typically predict a rugged landscape of interleaved islands of black hole and neutron star formation (figure 4). Massive stars are expected to blow up quite robustly, up to an initial mass of about $15 \mathrm{M}_{\odot}$. The fate of stars in the region $15-20 \mathrm{M}_{\odot}$ is highly uncertain; between $20 \mathrm{M}_{\odot}$ and $23 \mathrm{M}_{\odot}$, and above $28 \mathrm{M}_{\odot}$, explosions are particularly hard to achieve. Around $24 \mathrm{M}_{\odot}$ and $26 \mathrm{M}_{\odot}$ theory predicts "islands of explodability". Core-collapse supernova explosions may be possible for very massive progenitors that suffer from extreme mass loss during and after the main sequence.

The origin of this pattern of explodability has been traced to the core structure of supernova progenitors; quantitative measures for the explodability of massive stars have been proposed, such as the "compactness parameter" of O'Connor \& Ott (2011) and the two-parameter explosion criterion of Ertl et al. (2016). Qualitatively, the following structural features appear to determine the explodability:

- The onset of the explosion probably occurs when the oxygen burning shell is accreted. This shell interface is typically associated with a drop in density, so that the ram pressure onto the shock drops as the interface falls through the shock.

- If the oxygen shell is dense, the accretion does not drop significantly upon infall, and an explosion is less likely.

- The mass of the silicon core plays an ambiguous role. On the one hand, a protoneutron star from a massive silicon core leads to higher neutrino luminosities and mean energies and more heating. On the other hand, a massive silicon core typically comes with a denser oxygen shell around it, which tends to decrease explodability.

- No explosions are expected if the silicon core mass approaches the (unknown) maximum neutron star mass.

Predicting explosion properties - and not only "explodability" - is more challenging because such predictions depend more sensitively on how the neutrino-driven engine is approximated in a 1D simulation or an analytic framework. Explosion energies of the order of $10^{51} \mathrm{erg}$ can be obtained through phenomenological models (Ugliano et al. 2012, Pejcha \& Thompson 2015, Ertl et al. 2016, Sukhbold et al. 2016, Perego et al. 2015), but is the good match with observed explosion energies merely a result of the explicit or implicit calibration of model parameters? For example, Ertl et al. (2016) and Sukhbold et al. (2016) tune their model such as to reproduce the explosion energy of the SN1987A and the Crab supernova with progenitors of $15-20 \mathrm{M}_{\odot}$ and $9.6 \mathrm{M}_{\odot}$ respectively. Will self-consistent multidimensional supernova explosion models also reach these energies once we can extend them to sufficiently late times?

The approach of Müller et al. (2016a) sheds some light on this, because it seeks to extrapolate the findings from 
multidimensional simulations to predict explosion properties, instead of using observed supernovae as calibration points. This is accomplished by incorporating empirical scaling laws obtained from 3D simulations for the mass outflow rate of neutrino-heated matter and the growth of the explosion energy. Although this approach still has to deal with uncertainties in various parameters, it suggests that a reasonable distribution of explosion energies can be obtained with parameters that are roughly compatible with current 3D simulations.

For their standard set of parameters, Müller et al. (2016a) obtain a distribution of explosion energies peaking around $5 \times 10^{50} \mathrm{erg}$ with a tail reaching up to $2 \times 10^{51} \mathrm{erg}$, and masses of radioactive ${ }^{56} \mathrm{Ni}$ of a few $0.01 \mathrm{M}_{\odot}$. Kasen \& Woosley (2009) report that the typical observed value for explosions of Type II supernovae is around $9 \times 10^{50} \mathrm{erg}$. However, that is based on an observed magnitude limited sample (effectively the bright supernovae) and is not a complete, volume-limited sample. Hence a careful observational estimate of the true distribution of explosion energies is now required for a quantitative comparison. In line with long-time 2D simulations (Nakamura et al. 2015, Bruenn et al. 2016), they also find a weak trend for more massive progenitors to explode more energetically, which appears to be compatible with observations (Poznanski 2013).

The quantitative accuracy of phenomenological predictions is, of course, somewhat limited. By exploring parameter variations, Pejcha \& Prieto (2015) and Müller et al. (2016a) showed that there is considerable leeway in shifting the limit for the "explodability" of supernova progenitors without sacrificing decent overall agreement with observations in the typical explosion energy. By the same token, the explosion energy and the mass of radioactive ${ }^{56} \mathrm{Ni}$ can easily be shifted up or down by a factor of two by tweaking parameters within reasonable bounds. What proves robust, however, is the general shape of the landscape of explosion properties and the location (as opposed to the width) of the islands of neutron star and black hole formation.

Taken together, the various phenomenological approaches nonetheless suggest that the neutrino-driven mechanism may indeed be able to explain adequately the explosion properties of most core-collapse supernovae. This does not imply, of course, that the systematics of core-collapse supernova explosion properties are yet fully understood. The phenomenological approach is a shortcut through uncharted territory, and a range of 3D long-time simulations with detailed neutrino transport will be needed to back it up eventually.
4 Fraction of massive stars exploding as supernova in bins of $0.25 \mathrm{M}_{\odot}$ as predicted by the analytic supernova model of Müller et al. 2016a. A sizable fraction of massive stars is expected to form black holes already at moderate masses (at 15-16 $\mathrm{M}_{\odot}$ and $\left.18-19 M_{\odot}\right)$, while there are also two islands of explodability at $23-25 \mathrm{M}_{\odot}$ and 26-27 $\mathrm{M}_{\odot}$.

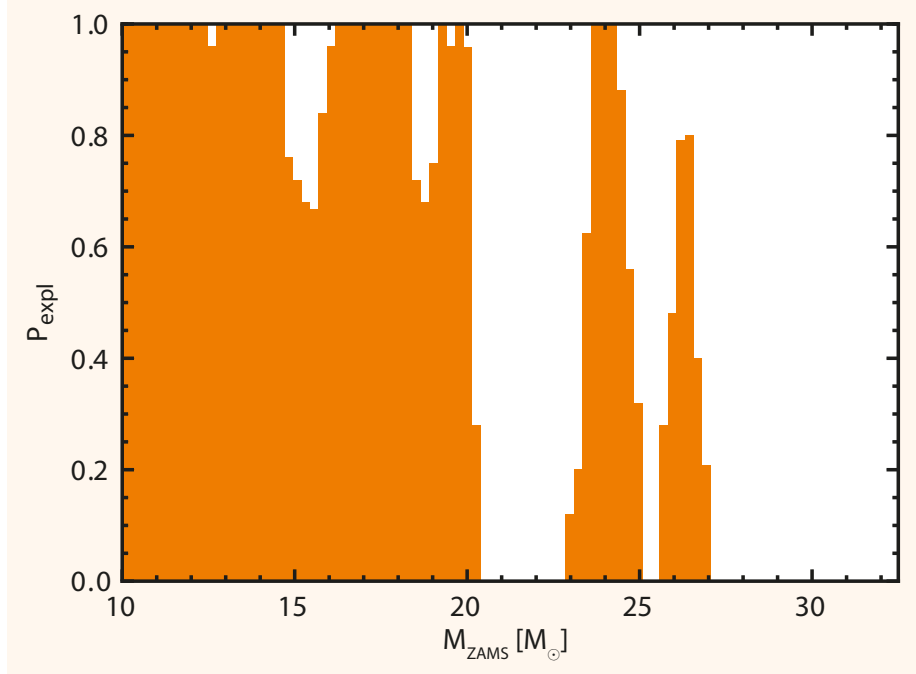

Even then, much of the input physics in supernova simulations - from the neutron star equation of state to the precise core structure of the progenitors - will remain subject to uncertainties that can only be eliminated by confronting the theoretical predictions with observations.

\section{Observing supernovae and progenitors}

The direct ways of testing the explosion models are to determine the mass ejected and energy of the explosion together with the amount of ${ }^{56} \mathrm{Ni}$ produced. The mass of the isotopes synthesized during hydrostatic burning during the last stages of stellar evolution (such as ${ }^{16} \mathrm{O},{ }^{20} \mathrm{Ne},{ }^{24} \mathrm{Mg}$ ) are also key tests (Jerkstrand et al. 2012). Determining ejecta masses and explosion energies require hydrodynamic models of the explosion and radiative transfer calculations to compare with supernova light curves and spectra. This, of course, introduces another model dependency on the results and depends on a reliable physical description of the hydrodynamic explosion (Pumo \& Zampieri 2011).

An even more fundamental test is to compare the explosion properties with progenitor star detections. For more than a decade, teams have been searching for direct detections of progenitor stars for the closest supernovae in the local universe (those closer than about 20Mpc), with results summarized in Smartt $(2009,2015)$. The progenitors of TypeII supernovae (the most common subtypes are called II-plateau) have been confirmed to be red supergiants. The TypeIIb supernovae show evidence of lower hydrogen and greater helium content in their ejecta and these have been confirmed to be yellow supergiants within binary systems (Van Dyk et al. 2011). Only one progenitor from a stripped envelope Ibc has been found, which is likely to be the explosion of a He star in a binary system. There are now about 20 detections of progenitor stars of core-collapse supernovae and around 30 upper limits within a volume-limited sample.

Perhaps the most interesting result is that the stellar masses inferred from the luminosities (based on stellar evolution models) do not follow the expected initial mass function from $8 \mathrm{M}_{\odot}$ up to $50-100 \mathrm{M}_{\odot}$. It's remarkable that there is a complete deficit of progenitors with luminosities correspond to an initial mass of $\mathrm{M} \leq 18 \mathrm{M}_{\odot}$. This lack of massive progenitors was first pointed out by Kochanek et al. (2008) and Smartt et al. (2009) and has grown ........ in statistical significance Thenitors of have been confirmed since. With the sample of 50 progenitor sites of nearby function would imply that we should have 13 high-mass progenitors above the luminosity limit of $\log L / L_{\odot} \approx 5.1 \mathrm{dex}$, but we have found noneor possibly one. The one case is SN2009ip, the nature of which is still debated (Mauerhan et al. 2013, Pastorello et al. 2013).

While there are arguments that the highmass progenitors may be giving TypeIbc supernovae and are not detected because they are hot and faint Wolf-Rayet stars, this appears unlikely (Eldridge et al. 2013). The observational constraints are in good qualitative agreement with the theoretical results of islands of explodability. Figure 5 shows a plot of the cumulative frequency distribution (CFD) of the progenitor masses and mass limits to date from Smartt (2015) and supplemented with more recent measurements of ASASSN-16fq (Kochanek et al. 2016) and SN2016gkg (Tartaglia et al. 2016). These include all Type II, IIb and Ib detections and upper limits, representing an extension of the volume- and time-limited sample compiled by Smartt (2015), with references therein. The CFD for the successfully exploded models of Müller et al. greater than $\log L / L_{\odot} \approx 5.1$ dex, which would core-collapse, the initial mass 
5 The cumulative frequency plot of the progenitor masses and upper limits to date ("data" line in orange) and the masses of the stellar models that do explode from Müller et al. 2016 ("explosion models" in cyan). A simple Salpeter IMF with alpha $=-2.35$ is shown with high mass truncated at 20,25 and $35 \mathrm{M}$

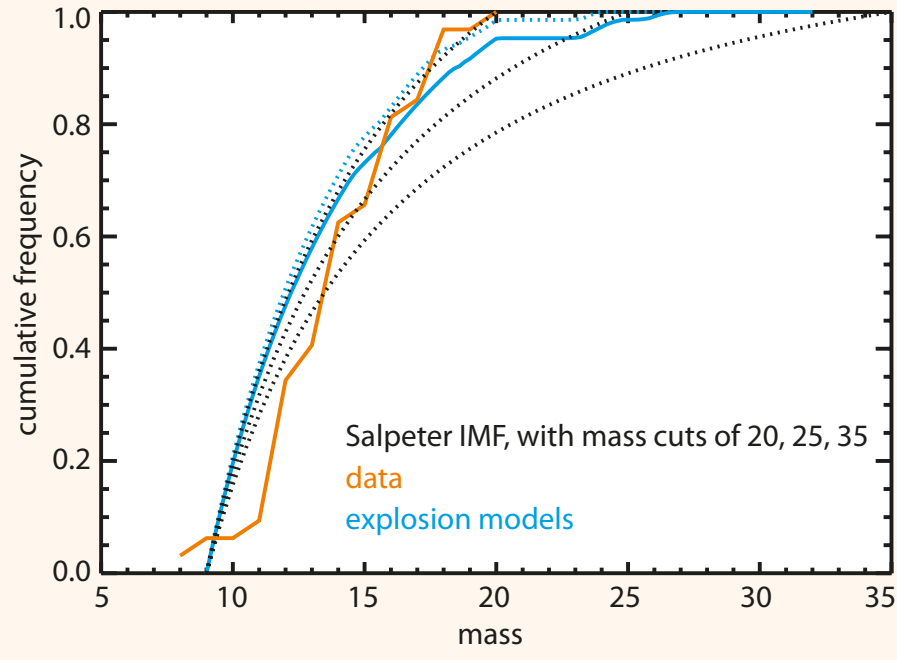

(2016a) is plotted for comparison and shows good agreement between the theory and data. The differences between the curves between about 12 and $14 \mathrm{M}_{\odot}$ are probably not significant given the uncertainty in the mass estimates of each progenitor detection of at least $2-4 \mathrm{M}_{\odot}$ (Smartt 2015, Smartt et al. 2009). In addition, many of the data points in this mass range ( 8 out of 20 ) are upper limits and hence a simple Kolmogorov-Smirnoff (KS) test would not be quantitatively reflective. Smartt (2015) used the maximum likelihood approach to cir-
$(0.00000$ "Other scenarios for extremely powerful explosions are proving elusive for observers" range (Anderson et al. 2012) and that their progenitors have evaded discovery to date because of the low numbers and relative faintness of Wolf-Rayet star progenitors.

\section{The most energetic explosions}

Theory and observations are thus on the way to converging on the question of which massive stars explode in the local universe. cumvent these two problems of the KS test, finding that a Salpeter IMF with minimum and maximim masses of $m_{\min }=9.5_{-2}^{+0.5}$ and $m_{\max }=16.5_{-2.25}^{+2.5}$ described the data well. Hence the major difference between the data and explosion models is the prediction of the high mass explodability islands in the region $23-27 \mathrm{M}_{\odot}$ (figure 4). It could be that some Type Ibc supernovae come from progenitors both in lower mass binaries (Eldridge et al. 2015) and in this higher mass ties. The observed explosion energies, for example, vary by two orders of magnitude from about $10^{50} \mathrm{erg}$ for low-energy events to $\sim 2 \times 10^{52} \mathrm{erg}$. The most energetic explosions span a range of types, from the "hypernova" explosions associated with gamma-ray bursts (Iwamoto et al. 1998) to superluminous supernovae (Nicholl et al. 2015) and high-energy TypeII events (e.g. Botticella et al. 2010, Utrobin et al. 2010). The standard paradigm of neutrino-driven explosions seems unable to explain the upper end of the supernova energy distribution because neutrino heating is a rather inefficient mechanism for tapping the neutron star binding energy of $\sim 10^{53} \mathrm{erg}$. Less than $10 \%$ of the emitted neutrino energy is reabsorbed in the heating region, and a large fraction of the energy input is spent merely to lift the ejecta out of the gravitational well of the neutron star. Phenomenological models of the neutrino-driven mechanism suggest that this leads to an upper limit of about $2 \times 10^{51} \mathrm{erg}$ for neutrino-driven explosions.

The most energetic core-collapse supernovae therefore require a different mechanism that operates only in a small subset of massive stars. The most likely contender is some kind of magnetorotational mechanism that taps the rotational energy of a rapidly rotating neutron star or, alternatively, a massive accretion disc around a nascent black hole (Kasen \& Bildersten 2010, Woosley 2010, Dexter \& Kasen 2013, Akiyama et al. 2003). While the available energy reservoir (a few $10^{52} \mathrm{erg}$ ) is smaller than the binding energy of a neutron star that feeds the neutrino emission, sufficiently strong magnetic fields may be able to transfer it efficiently to the ejecta. Magnetohydrodynamic effects also provide a plausible explanation for collimated jet outflows in the gamma-ray bursts (Woosley \& Bloom 2006) associated with some hypernovae.

Especially from the viewpoint of supernova and stellar evolution theory, this scenario remains subject to many more imponderables than the vast majority of "ordinary" core-collapse supernovae. Other scenarios for extremely powerful explosions - like so-called pair-instability supernovae - are well-rooted in theory, but prove more elusive for observers. Nonetheless, our emerging picture of the connection between supernovae and their progenitors remains incomplete without them. •

\section{AUTHORS}

Bernhard Müller, Astrophysics Research Centre, Queen's University Belfast, UK, and Monash Centre for Astrophysics, Monash University, Australia; b.mueller@qub.ac.uk. Stephen J Smartt, Astrophysics Research Centre, Queen's University Belfast, UK

\section{ACKNOWLEDGMENTS}

SJS acknowledges funding from the (FP7/20072013)/European Research Council Grant agreement no. 291222 and Science and Technology Facilities Council grants ST/1001123/1 and ST/L000709/1. Figure 3 is based on work supported by the STFC DiRAC HPC Facility (DiRAC Data Centric system, ICC Durham), the National Computational Infrastructure (Australia) and the Pawsey Supercomputing Centre (University of Western Australia).

\section{REFERENCES}

Abdikamalov E et al. 2016 Mon. Not. R. Astron. Soc. 4613864

Akiyama S et al. 2003 Astroph. J. $\mathbf{5 8 4 9 5 4}$

Anderson JP et al. 2012 Mon. Not. R. Astron. SoC 4241372
Baade W \& Zwicky F 1934a Proc Nation. Acad Sci. 20259

Baade W \& Zwicky F 1934b Proc. Nation. Acad.

Sci. 20254

Blondin JM et al. 2003 Astroph. J. 584971

Botticella MT et al. 2010 Astroph. J. Letts 717 L52 Bruenn SW et al. 2016 Astroph. J. 818123 Burrows A et al. 1995 Astroph. J. 450830

Colgate SA \& White RH 1966 Astroph. J. 143626 Couch SM \& Ott CD 2013 Astroph. J. Letts 778 L7 Couch SM et al. 2015 Astroph. J. Letts 808 L21

Dexter J \& Kasen D 2013 Astroph. J. 77230 Eldridge JJ et al. 2013 Mon. Not. R. Astron. Soc. 436774

Eldridge JJ et al. 2015 Mon. Not. R. Astron. Soc 4462689

Ertl T et al. 2016 Astroph. J. 818124

Fraser Met al. 2011 Mon. Not. R. Astron. Soc. 417141 Fryer CL \& Kalogera V 2001 Astroph. J. $\mathbf{5 5 4} 548$

Fryer CL et al. 2012 Astroph. J. 74991

Hanke FM et al. 2013 Astroph. J. 77066

Heger A et al. 2003 Astroph. J. 591288

Herant M et al. 1992 Astroph. J. 395642

Iwamoto Ket al. 1998 Nature 395672

Janka H-T 2012 Ann. Rev. Nucl. Part. Sci. 62407
Janka H-T et al. 2016 Ann. Rev. Nucl. Part Sci. 66341 Jerkstrand A et al. 2012 Astron. Astrophys. 54628 Kasen D \& Bildsten L 2010 Astroph. J. 717245 Kasen D \& Woosley SE 2009 Astroph. J. 7032205 Kitaura FS et al. 2006 Astron. Astrophys. 450345 Kochanek CS et al. 2008 Astroph. J. 6841336 Kochanek CS et al. 2016 arXiv:160900022K Lentz EJ et al. 2015 Astroph. J. Letts 807 L3 Liebendörfer M et al. 2001 Phys. Rev. D 63 103004:1

Lindquist RW 1966 Ann. Phys. 37487

Mauerhan JC et al. 2013 Mon. Not. R. Astron. Soc 4301801

Maund JR et al. 2005 Mon. Not. R. Astron. Soc $364\llcorner 33$

Maund JR et al. 2014 Mon. Not. R. Astron. Soc 4381577

Melson T et al. 2015a Astroph. J. Letts 808 L42 Melson T et al. 2015b Astroph. J. Letts 801 L 24 Müller B 2015 Mon Not R Astron Soc 453287 Müller B \& Janka H-T 2015 Mon. Not. R. Astron Soc. 4482141

Müller B et al. 2016a Mon. Not. R. Astron. Soc 460742

Müller B et al. 2016b Astroph. J. 833124
Nakamura Ket al. 2015 Proc Astron. Soc Japan 67107 Nicholl M et al. 2015 Mon. Not. R. Astron. Soc 4523869 O'Connor E \& Ott CD 2011 Astroph. J. 73070 Pastorello A et al. 2013 Astroph. J. 767 Pejcha O \& Prieto JL 2015 Astroph. J. 806225 Pejcha O \& Thompson TA 2015 Astroph. J. 80190 Perego A et al. 2015 Astroph. J. 806275 Poznanski D 2013 Mon. Not. R. Astron. Soc. 436322 Pumo ML \& Zampieri L 2011 Astroph.J. 74141 Rampp M \& Janka H-T 2000 Astroph. J. Letts. $539\llcorner 33$

Smartt SJ 2009 Ann. Revs Astron. Astrophys. 4763 Smartt SJ 2015 Pub. Astron. Soc. Australia 3216 Smartt S et al. 2009 Mon. Not. R. Astron. Soc. 3951409

Sukhbold T et al. 2016 Astroph. J. 82138 Tartaglia Let al. 2016 arXiv:161100419T Ugliano M et al. 2012 Astroph. J. 75769 Utrobin VP et al. 2010 Astroph. J. Letts. 723 L89 Van Dyk S et al. 2011 Astroph.J. 74128

Woosley SE 2010 Astroph. J. Letts. 719 L204 Woosley SE \& Bloom JS 2006 Ann. Revs Astron Astrophys. 44507 